\title{
Extraction of Road Traffic Signs using Similarity of Adjacent Color Information
}

\author{
Chunghsin LIAO Hiromitsu HAMA \\ Graduate School of Engineering, Osaka City University \\ 3-3-138, Sugimoto, Sumiyoshiku, Osaka558-8585, Japan \\ E-mail: liao@sys.info.eng.osaka-cu.ac.jp,
}

\begin{abstract}
This research is aiming at creation a visual supplement system. In the current researches of automatic extraction system of road traffic signs, the noise due to the variation of illumination condition, and the obscure image due to shielding affected negatively about the correct extraction of road traffic signs. In this research, robust characters were applied in the extraction of road traffic signs, adding with the new alternative method, to improve the road traffic sign recognition system. Audio warning and visual expression were added to remind the driver for a better warning.
\end{abstract}

\section{Introduction}

With the increase of traffic in recent years, the increase of traffic accidents induces a profound problem of the society. It also becomes a primary issue of every country. The major cause of traffic accident comes from the negligence of driver about the information in front, such as road traffic signs, obstacles, other vehicles, etc. To prevent traffic accident, road traffic signs play an important role. The purpose of this design is to enhance drivers' attention about the road traffic signs in front. Due to their own psychological and physical conditions, drivers frequently overlook the traffic signs before them and cause traffic accidents. Thus, this research studied the cause of the accidents and aims to provide drivers suitable instructions to prevent traffic accidents out of dangerous actions and negligence.

The color image taken by a car-carried camera was analyzed and the analysis result of road traffic signs was prompted for the driver's attention. Under a continued ignorance of the warning from the driver, the system will take over the control of the vehicle. Figure 1 shows expression method. To establish a safe, comfortable driving environment and to make the system realization, high precision and high speed recognition is necessary.

\section{System Component}

Providing drivers safe and accurate information is very important. In the design of this system, the automatic recognition of traffic sign, processing time, and the robust to attend to surrounding changes must be carefully considered. To realize the system, a proposal of extraction Algorithm of road traffic sign was proposed. In this proposal, the color image taken from camera will be performed the HSL (H:Hue, S:Saturation, L:Light) conversion first [1][2][3]. HSL is a system which will not have a significant coloration change in outdoor environment having significant illumination variation.

The useless color information of traffic sign was first removed then certain seed pixels were assigned to probable regions of road traffic signs left. With this method, the regions of road traffic sign can be extracted.

The response duration of traffic sign extraction system is calculated from the distance theory of traffic regulation that when driver found there was danger or obstacle ahead, the driver stepped on the brake and induced the total stop of vehicle. Driver should be accurately and suitably prompted with this 
period. Figure 2 shows relation of braking distance and speed.

\section{Road Traffic Sign Extraction Algorithm}

In the past, gray scale camera was used to recognize road traffic signs. The image abstracted has an outer white contour, which has a high light value. Road traffic signs were thereby possibly to be extracted. However, when the outer counter breaks or the white line fades or blurs out, it is difficult to extract the road traffic signs.

And by using threshold or mode methods, the expected region and the background were separated to extract the road traffic sign. However, these methods are not suitable in outdoor environment which has a high variation of illumination. The solution is explained as follows.

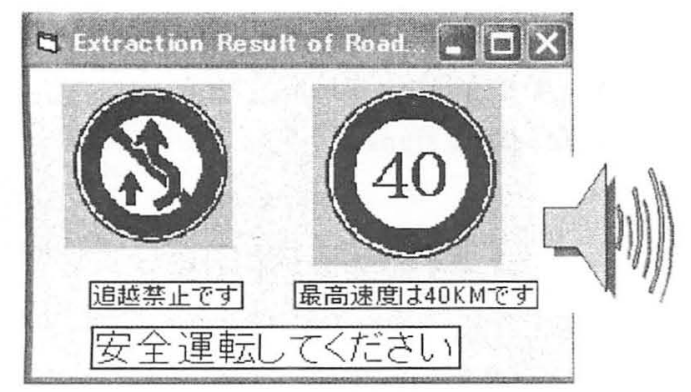

Fig.1 Expression method. (Audio warning and Visual expression)

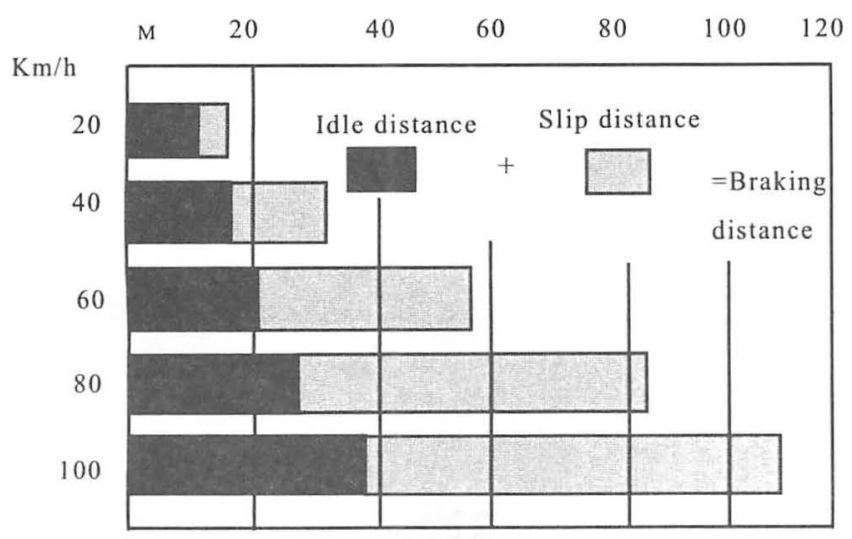

Fig2 Relation of braking distance and speed

\subsection{HSL Coloration Method}

The images obtained from camera consist of 8 bits of
RGB, and the size is $320 \times 240$ pixels. It is difficult to extract road traffic sign from the simple RGB configurations, especially at night or in reverse light condition. At night, the image is dark, we need to remove the dark part of the image, expressing by equations (1), (2) and (3) as follows:

$$
\begin{aligned}
& H=\tan ^{-1}\{\sqrt{3}(G-B) /[(R-G)+(R-B)]\} \\
& S=1-\min (R, G, B) / L \\
& L=(R+G+B) / 3
\end{aligned}
$$

\subsection{The Removal of Colors and Shapes Out of Road Traffic Signs}

In accordance with law of road traffic sign, The blue and yellow colors of road traffic sign provide people the effects of clear judgment and alertness. Red, blue and yellow are the basic colors. The shapes allowed include circle, rhombus, and triangle. If we can use the above two features effectively then we can effectively process road traffic signs.

\subsubsection{Probable Regions of Road Traffic Signs}

First, we focus on the colors not used in road traffic signs [4]. These useless pixels were removed first[5][6]. If we performed in the reversed direction, to find the possible pixels in road traffic signs, then these possible pixels may not be effectively and accurately extracted because of the problem of variation of illumination. 4 points each were taken as sample pixels from the colors of red, blue, and yellow. Then, on the center of the three-parameter normalization spectrum of HSL, taking the central value as the center, these samples were fitted into a certain interval as the final values. Figure 3, 4, 5 show the hue, saturation, and light normalization spectrum of road traffic signs.

About the sample number of the images, for the red color, 250 images were taken on cloudy days and 200 images were taken on sunny days. Figure 6 shows the probable regions of road traffic signs from HSL color information. 


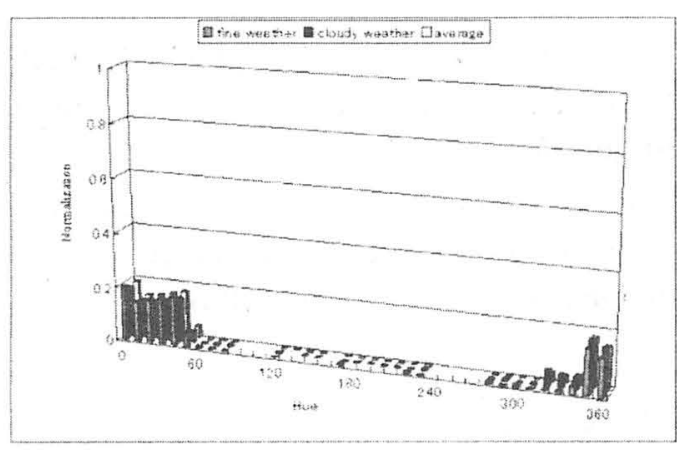

(a) Red

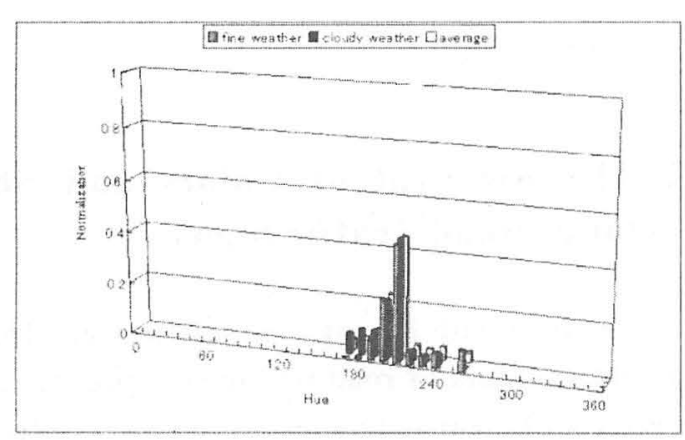

(b) Green

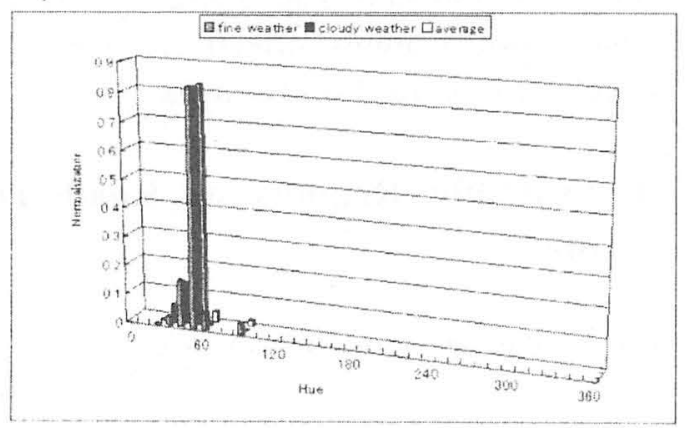

(c) Yellow

Fig.3 Hue normalization spectrum of road traffic signs

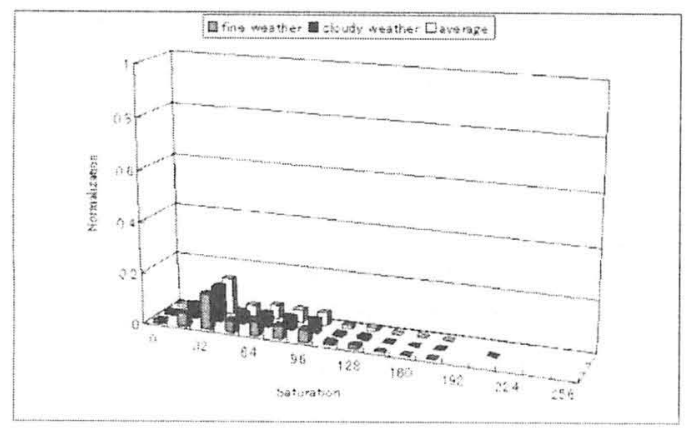

(a) Red

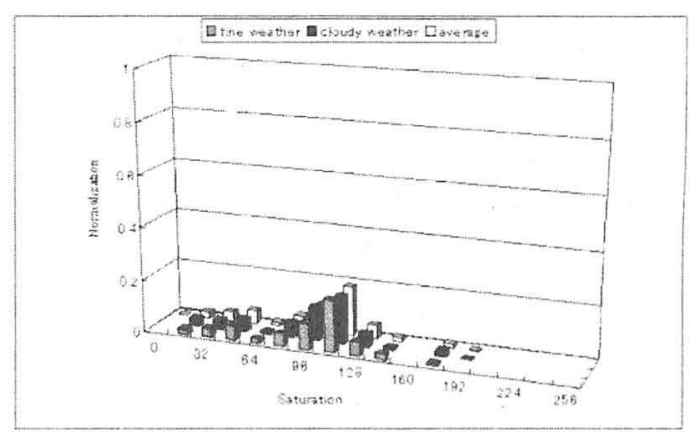

(b) Green

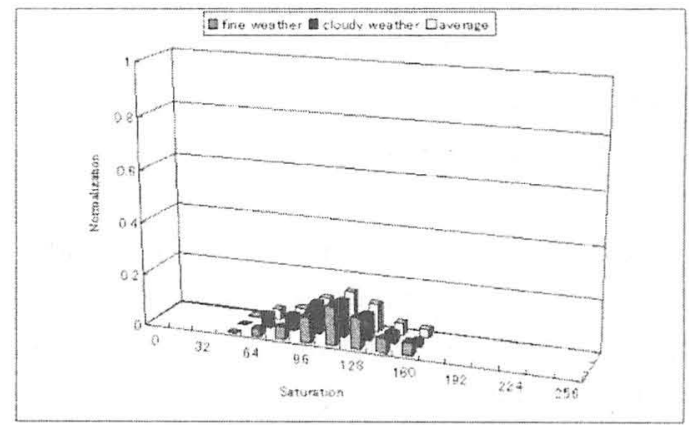

(c) Yellow

Fig.4 Saturation normalization spectrum of road traffic signs

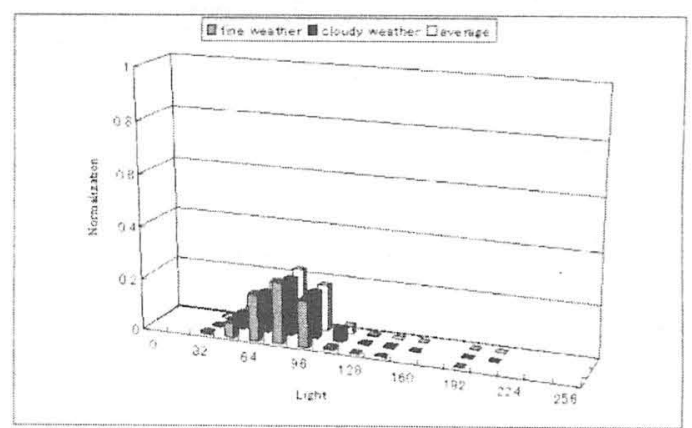

(a) Red

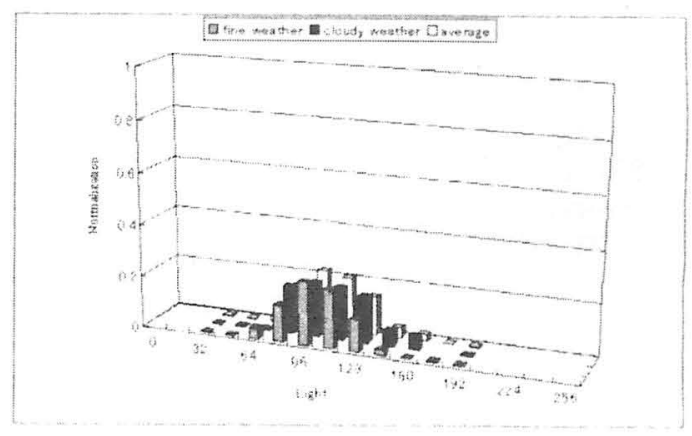

(b) Blue 


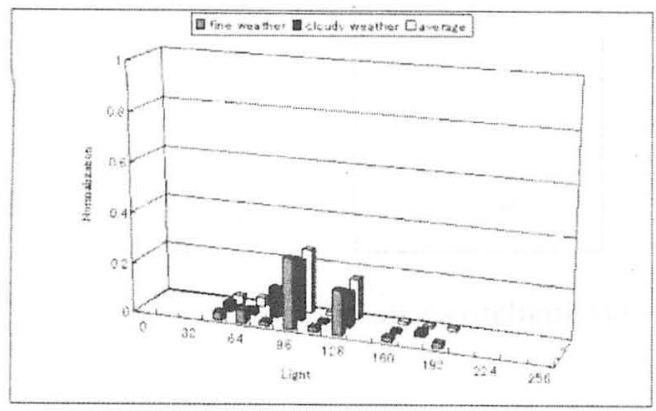

(c) Yellow

Fig.5 Light normalization spectrum of road traffic signs

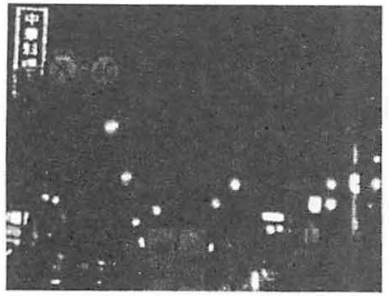

(a) Input image

(Night environment)

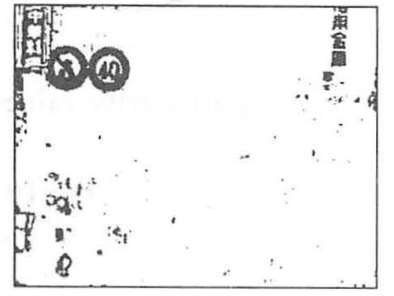

(b) Probable regions of road traffic sign
Fig.6 Probably regions by HSL color information

\subsection{Seed Pixel Tracking}

In physics, when light projects on object, the reflection will have equilibrium diffusion on the object. It was not influenced by the materials. Traffic sign will express the same quality in the principle. There should be no big difference in nearby pixel, unless they are different colors.

\subsubsection{Similarity Value}

From the probable regions of road traffic sign, the seed point $A$ was selected. The adjacent pixel $B$ was also selected. The similarity value of $\overline{A B}$ can be calculated from equation (4).

$$
\begin{aligned}
& \overline{A B}=\sqrt{W_{h}\left(h_{i}-h_{j}\right)^{2}+W_{s}\left(s_{i}-s_{j}\right)^{2}+\left(W_{v}\left(l_{i}-l_{j}\right)^{2}\right.} \\
& W_{h}=0.5 \quad W_{s}=0.3 \quad W_{l}=0.2
\end{aligned}
$$

Here, $h s l$ is the HSL value of seed $A$ and $B$; $W$ is the weight coefficient. Starting from seed pixel $A$, by counterclockwise direction, the adjacent eight neighborhood were calculated their similarity value. If they meet the preset similarity value, then they are taken as the same object. The algorithm is described as follows:

A) In the probable regions of road traffic sign, assigned seed pixels by suitable interval.

B) From the seed pixel, starting from the right side, calculating by counterclockwise direction to search the adjacent eight-neighborhood.

C) If the pixel is within the preset similarity value with the starting point, then it is taken as the same object.

D) If the adjacent eight-neighborhood pixel in the each direction is farther than the present similarity value, then the search is ended.

Here, a practical example was illustrated. In Figure 7(a) seed pixels were assigned by a certain interval. After the application of the aforesaid algorithm, Figure 7(b) was sowed as the result.

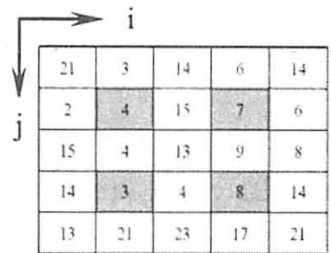

(a) Assign seed pixels (b) by suitable interval

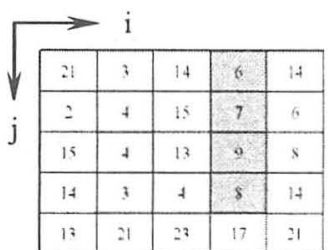

(b) Result by proposed algorithm (similarity value $=2$ )
Fig.7 An example of proposed algorithm

\subsubsection{Labeling by Adjacent Region Color Information}

In Figure $7(\mathrm{a})$ at coordinate $(1,1),(1,3),(3,1),(3,3)$ a seed pixels are given. Figure $7(\mathrm{~b})$ let $\Omega$ be the probable region of road traffic sign and have size $i \times j$. First, assume the preset similarity value is 2 . Then, according to the aforesaid, there are two objects. We call them object $X$ and object $Y$. The area is the total pixels with each object. Therefore, the area of object $X$ is 6 and the area of object $Y$ is 4 .

If the preset similarity value is 4 , then there is only one object. The area calculating from equation (4) is 10. In the Domogram Law of Set Theory, in the two objects $X$ and $Y, X \subset Y$ means "All the element of $X$ can be found in $Y$ " and visa versa. If $X \subset Y$ and $X \supset Y$ are both true, then $X=Y$. That means, the two objects are merged into one object. 
For one object, which means $X$ and $Y$ have common region, no matter seed was assigned in which place, at a certain similarity value, the area of the object is the same.

Let the similarity value is 1 . The object obtained from P1 was shown in Figure 9 and the object obtained from P2 was shown in Figure 10. The preset similarity value was increased by an increment of 1 and the same experiments were performed in P3 and P4. When the preset similarity value exceeds than a certain number, the area of object increases greatly. The increased area is related with the background colors.

We verify the white region with the same method. The seed points in white region were P5 and P6 and the result was shown in Figure 12. It shows that the two objects formed by using seeds in different color regions did not overlap. That means that the object formed by seed from red region will not affect object formed by seed from white region. This proves the effectiveness of the seed method. The same objects formed were linked with the same label and different objects formed were linked with different label.

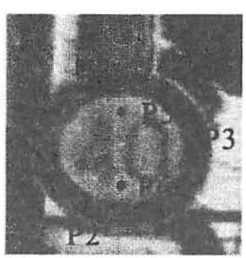

Fig. 8 An image of preparing experiment

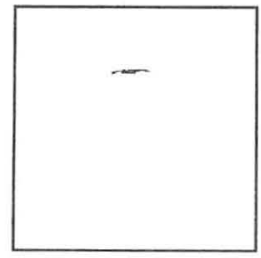

(a) Similarity value $=1$

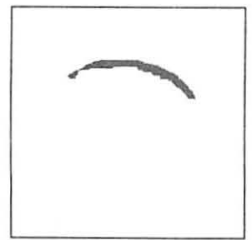

(c) Similarity value $=3$

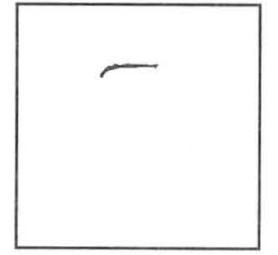

(b) Similarity value $=2$

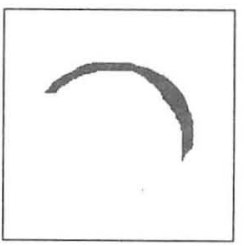

(d) Similarity value $=4$

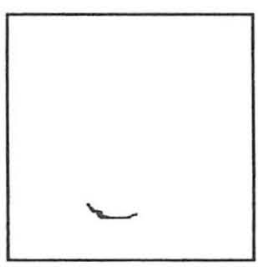

(a) Similarity value $=1$

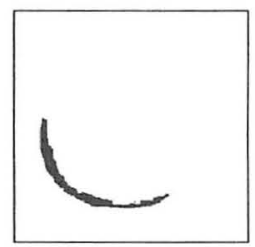

(c) Similarity value $=3$

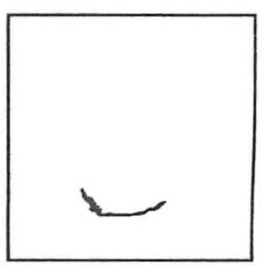

(b) Similarity value $=2$

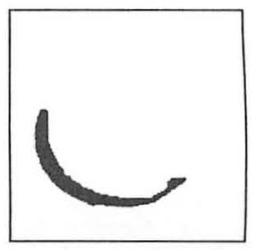

(d) Similarity value $=4$
Fig.10 Region from P2

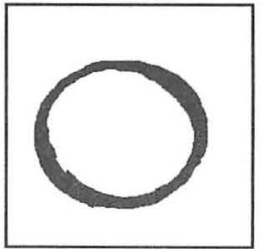

(a) Similarity value $=5$

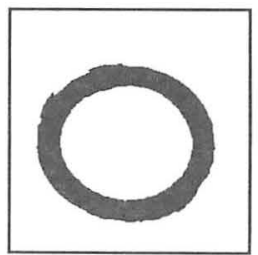

(c) Similarity value $=7 \sim 15$

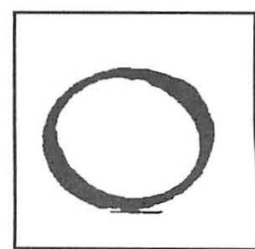

(b) Similarity value $=6$

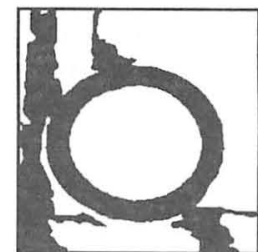

(d) Similarity value $=16$
Fig.11 Result when common region from $\mathrm{p} 1$ and $\mathrm{p} 2$

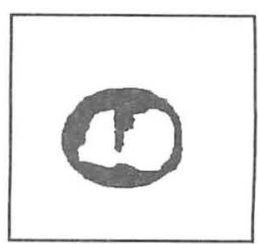

Fig. 12 Result from $\mathrm{p} 5$ and $\mathrm{p} 6$ (Similarity value $5 \sim 11$ )

Fig.9 Region from P1 


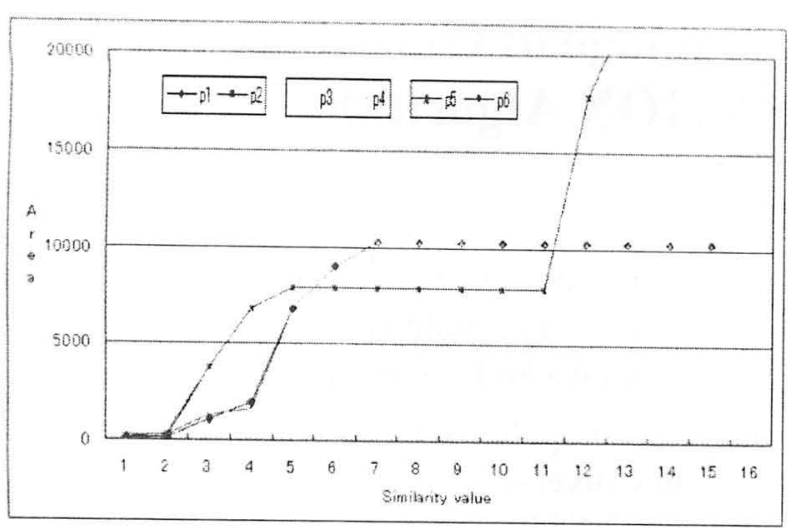

Fig.13 Result from P1, P2 and P3, P4 and P5, P6

\section{Experimental Result}

To verify the practicality of this research, a road test was performed in the Abiko streets of Osaka city. Images were taken from the passenger seat by CCD video camera. The image on the video tape was then read out by an interval of 0.1 second and stored in the hard drive of a computer for subsequent extraction and procession.

A computer equipped with a Pentium $4,2.6 \mathrm{GHz}$ CPU was used in the extraction of 3480 images. The extraction rate and processing time were showed in Table 1. The calculation method of extraction rate is the correct extraction from all images within braking distance. The better extraction rate showed in the result proves that the method in this research is better than past methods.

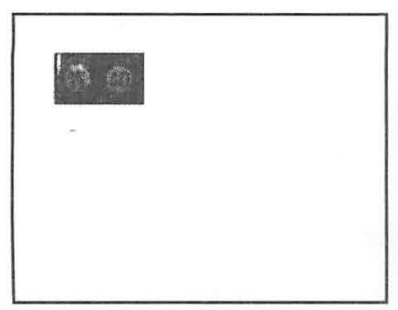

Fig.14 Extraction result

Fig.15 An example of extraction succeeded and extraction failed

(Evening glow)

\section{Conclusion}

The extraction algorithm of road traffic sign of this research proved excel results in environment with illumination variation and when applied in real road situation. It can be used as a visual supplement system. In the algorithm of this proposal, specific colors of traffic signs were used in recognition. Thereby the smaller recognition region enhances the processing efficiency.

There are some situations that the extraction could not be successful, the reasons are listed as follows:

A) When the colors of the background are the same as the road traffic sign, there is difficulty in correct extraction.

B) The color fading due to aging induces a big difference between the original color and the actual color of road traffic sign.

C) The shielding of road traffic sign by obstacle.

In driver reminding, it needs more endeavors in many ways if we would like to connect this supplement system with eye camera and provide cue issues to road traffic signs which are necessary and the driver does not pay attention to.

Table 1 Comparison between past method and proposed method

\begin{tabular}{|l|c|c|c|}
\hline & $\begin{array}{l}\text { Using HSL } \\
\text { Only }\end{array}$ & $\begin{array}{l}\text { RGB } \\
\text { Subtraction }\end{array}$ & $\begin{array}{l}\text { HSL and Proposed } \\
\text { method }\end{array}$ \\
\hline $\begin{array}{l}\text { Extraction } \\
\text { rate }\end{array}$ & $83.43 \%$ & $92.54 \%$ & $97.82 \%$ \\
\hline $\begin{array}{l}\text { Processing } \\
\text { time }\end{array}$ & $0.22 \mathrm{sec}$ & $0.16 \mathrm{sec}$ & $0.28 \mathrm{sec}$ \\
\hline
\end{tabular}

\section{References}

[1] S. Azami, S. Katahara, and M. Aoki, "Route guidance sign identification using 2-D structural description," Proc. 1996 IEEE Symp. On Intelligent Vehicles, pp.153-158, 1996.

[2] E. Ichihara, H. Takao, and Y. Ohta, "Visual assistance for drivers using roadside cameras," Proc. 1999 IEEE Int. Conf. On Intelligent Transportation Systems, pp.170-175, Tokyo, Japan, Oct. 1999.

[3] Y.Y. Tang, S.W. Lee, and C.Y. Suen, "Automatic document processing: A survey," Pattern Recognition., vol.29, no.12, pp.1931-1952, 1996.

[4] G. Salgian and D.H. Ballard, "Visual routines for autonomous driving, " Proc. $6^{\text {th }}$ Int. Conf. On Computer Vision, pp.876-882, 1998.

[5] H. Kawakami, T. Ikeda, and J. Tajima, "Road sign detection using information about color and shape," IEICE Technical Report, PRU92-29, 1992

[6] G. Piccioli, E. De Micheli, P. Parodi, and M. Campani, "A robust method for road sign detection and recognition," Image and Vision Computing, vol.14, pp.209-223, 1996. 